OPEN ACCESS

Edited by:

Paolo M. Cattaneo,

University of Melbourne, Australia

Reviewed by:

Roberto Savignano,

Loma Linda University, United States

Jialiang Wang,

University of Wisconsin-Madison,

United States

*Correspondence:

Tarek M. Elshazly

tarek.m.elshazly@gmail.com

elshazly@uni-bonn.de

tORCID:

Tarek M. Elshazly orcid.org/0000-0002-3219-9048

${ }^{*}$ These authors have contributed equally to this work and share last authorship

Specialty section:

This article was submitted to

Biomaterials,

a section of the journal

Frontiers in Materials

Received: 13 October 2021 Accepted: 07 December 2021 Published: 28 January 2022

Citation:

Elshazly TM, Keilig L, Alkabani Y, Ghoneima A, Abuzayda M, Talaat W,

Talaat $S$ and Bourauel CP (2022) Potential Application of 4D Technology in Fabrication of Orthodontic Aligners.

Front. Mater. 8:794536.

doi: $10.3389 /$ fmats.2021.794536

\title{
Potential Application of 4D Technology in Fabrication of Orthodontic Aligners
}

Tarek M. Elshazly ${ }^{1 * \dagger}$, Ludger Keilig $^{1,2}$, Yasmine Alkabani ${ }^{3}$, Ahmed Ghoneima ${ }^{4}$,
Moosa Abuzayda ${ }^{5}$, Wael Talaat ${ }^{6,7}$, Sameh Talaat ${ }^{1,8 \neq}$ and Christoph P. Bourauel ${ }^{1 \neq}$

${ }^{1}$ Oral Technology Department, Dental School, University Hospital Bonn, Bonn, Germany, ${ }^{2}$ Department of Dental Prosthetics, Propaedeutics and Materials Science, Dental School, University Hospital Bonn, Bonn, Germany, ${ }^{3}$ Restorative and Dental Materials Department, National Research Centre, Giza, Egypt, ${ }^{4}$ Department of Orthodontics, College of Dental Medicine, MBRU, Dubai, United Arab Emirates, ${ }^{5}$ Department of Prosthodontics, College of Dental Medicine, MBRU, Dubai, United Arab Emirates, ${ }^{6}$ Department of Oral and Craniofacial Health Sciences, College of Dental Medicine, University of Sharjah, Sharjah, United Arab Emirates, ${ }^{7}$ Department of Oral and Maxillofacial Surgery, Faculty of Dentistry, Suez Canal University, Ismailia, Egypt, ${ }^{8}$ Department of Orthodontics, Future University in Egypt, Cairo, Egypt

Objectives: To investigate and quantify forces generated by three-dimensional-printed aligners made of shape memory polymers (four-dimensional [4D] aligner).

Methods: Clear X v1.1 material was used in this study. On a custom-made typodont model, correction of maxillary central incisor (tooth 21) malposition by 4D aligners with thicknesses of 0.8 and $1.0 \mathrm{~mm}$ was measured by superimposition of subsequent scans. Maximum deflection forces generated by foil sheet specimens were measured at different temperatures in three-point bending (3-PB) tests. In a biomechanical system (orthodontic measurement and simulation system [OMSS]), forces generated on movements of tooth 21 by the 4D aligners were measured at different temperatures.

Results: 4D aligners succeeded to achieve a significant tooth movement $(2.5 \pm 0.5 \mathrm{~mm})$ on the typodont, with insignificant difference between different thicknesses. In the 3-PB test, the maximum deflection forces measured at $20,30,37,45$, and $55^{\circ} \mathrm{C}$, were $3.8 \pm 1.1$, $2.5 \pm 0.9,1.7 \pm 0.6,1.0 \pm 0.4$, and $0.5 \pm 0.4 \mathrm{~N}$, respectively. Forces delivered on palatal displacement of tooth 21 at 37,45 , and $55^{\circ} \mathrm{C}$ by 0.8 -mm aligners were $0.3 \pm 0.1,0.2 \pm 0.1$, and $0.7 \pm 0.2 \mathrm{~N}$, respectively, whereas those by 1.0 -mm aligners were $0.3 \pm 0.1,0.3 \pm 0.1$, and $0.6 \pm 0.2 \mathrm{~N}$, respectively. A good concordance with movement on the typodont model was shown in OMSS.

Conclusion: An initial study of 4D-printed aligner shows its ability to move a tooth by biocompatible orthodontic forces, after a suitable thermal stimulus within the oral temperature range.

Keywords: clear aligners, shape memory polymers, biomaterial, biomechanics, tooth movement, 4D printing, orthodontics 


\section{HIGHLIGHTS}

- Rate-limiting staging of conventional aligners consumes time and materials.

- Orthodontic aligners can be made of different polymers.

- Four-dimensional (4D) technology is a three-dimensional printing of polymeric shape memory material.

- Introduction of $4 \mathrm{D}$ technology in the field of orthodontic aligners is highly innovative.

- 4D aligners can move teeth by biocompatible forces.

\section{INTRODUCTION}

Aesthetics is one of the major demands of patients who seek dental treatment. Major problems facing patients of orthodontic treatment are the bad appearance of metallic orthodontic braces, keeping good oral hygiene, and the long treatment time (Elshazly et al., 2021). In the last decade, treatment by orthodontic aligners has shown growing interest (Ercoli et al., 2014; Ojima and Kau, 2017). The aligners gain great superiority in aesthetic, comfort, and oral hygiene, over fixed braces, even the ceramic and lingual ones, owing to the fact that they are clear and almost invisible, as well as the ability to remove them during eating, brushing, and flossing (Mehta and Mehta, 2014). Additionally, with clear aligners, treatment time and chair time are reduced in nonextraction cases, sometimes by more than the half, compared with fixed braces (Tamer et al., 2019).

Conventional aligners are made from different types of polymers (Ercoli et al., 2014), such as polyvinyl material, polyvinyl chloride, polyethylene terephthalate glycol (Thukral and Gupta, 2015), polypropylene, polyester, and polyurethane (Momtaz, 2016). The functioning principle of such appliances is based on limited movement of each tooth through a programmed deviation between the real tooth position and a setup position. The programmed geometry of the aligner's stent then defines the new tooth position and the amount of movement to be performed (Boyd and Waskalic, 2001). In most of the popular aligner systems in the market, each aligner is designed to move a tooth within the restriction of 0.2 to $0.3 \mathrm{~mm}$ for translations and $1^{\circ}$ to $3^{\circ}$ for rotations, for that it is worn approximately 14 days, and then it should be changed with its successor (Kwon et al., 2008; Thukral and Gupta, 2015; Elkholy et al., 2016). This stepwise staging of conventional aligners leads to time and material consumption and consequently high cost of the treatment (Martorelli et al., 2013; Ercoli et al., 2014; Elshazly et al., 2021)

With the introduction of the technology of digital scanning, three-dimensional (3D) printing, and CAD-CAM, the orthodontic aligner became more precise. Nonetheless, investigators are continuously working on improving the efficiency of the treatment. Many optimizations, innovations, and advances are aiming to facilitate the process and to reduce the time and the cost (Phan and Ling, 2007; Morton et al., 2017; Elshazly et al., 2021). Several researchers are focusing on two main drawbacks: the shortcomings of conventional materials and the biological consideration of tooth movement. Numerous methods have been introduced to biologically accelerate tooth movement (Ojima and Kau, 2017). On the other side, introduction of new aligner material also draws attention (Silverman and Cohen, 1969; Choi and Kim, 2005; Lai and Rule, 2020; Elshazly et al., 2021).

Shape memory polymers (SMPs) are one of the novel materials to be recently introduced into the field of dentistry, particularly for orthodontic applications (Mahmood et al., 2019; Elshazly et al., 2021). They provide great potential for applications in medical materials (Lendlein and Langer, 2002; Zhang et al., 2009; Meng and Li, 2013). In a study by Jung et al. (Jung and Cho, 2010) and another by Nakasima et al. (1991), SMPs were used in fabrication of orthodontic wires. Choi and Kim (2005) registered a patent about a tray-type appliance made of SMPs used for teeth alignment. Also, Lai and Rule (2020) registered another patent about an orthodontic appliance having a continuous shape memory recovery. Through this shape memory property, it can store a large number of geometries throughout the orthodontic treatment. Recently, Elshazly et al. (2021) reported a new orthodontic aligner system based on thermoresponsive shape memory polyurethane-based thermoplastic material, showing the ability of one aligner to recover its shape through three steps of material treatment and consequently conduct stepwise tooth movement in a way that one aligner may be able to replace three subsequent conventional aligners. Despite the mentioned reports, yet, there is lack of data in literature about the application of SMPs in the field of orthodontic aligners.

The technology of four-dimensional printing ( $4 \mathrm{D}$ printing) is based on the $3 \mathrm{D}$ printing of shape memory materials. Clearly, 4Dprinted parts have the ability to change shape with time (the fourth dimension), upon given environment conditions (Pei et al., 2020). With the continuous development of SMPs, new 4D printing applications within the product design industry are expected to grow (Nam and Pei, 2020). We proposed in our scientific project to introduce the $4 \mathrm{D}$ technology in the fabrication of orthodontic aligners.

In this study, on a custom-made typodont model, correction of malposition of tooth 21 by $4 \mathrm{D}$-printed aligners with thicknesses of 0.8 and $1.0 \mathrm{~mm}$ was measured by superimposition of obtained and initial scans. Also, forces generated on $2-\mathrm{mm}$ vertical threepoint bending (3-PB) tests were measured at different temperatures. Additionally, forces and movements delivered by the $4 \mathrm{D}$ aligners were quantified using an orthodontic measurement and simulation system (OMSS).

\section{MATERIALS AND METHODS}

\section{Typodont}

A custom-made typodont model was fabricated from resin (Technovit 4004; Kulzer, Wehrheim, Germany) and acrylic teeth (Frasaco, Tetnang, Germany). The upper left central incisor (tooth 21) was separated and kept movable by being embedded in pink wax placed in the model, while the other teeth were fixed by the resin (Figure 1). The fully aligned model was scanned (scan 0) using a 3D lab scanner (D2000; 3Shape, 

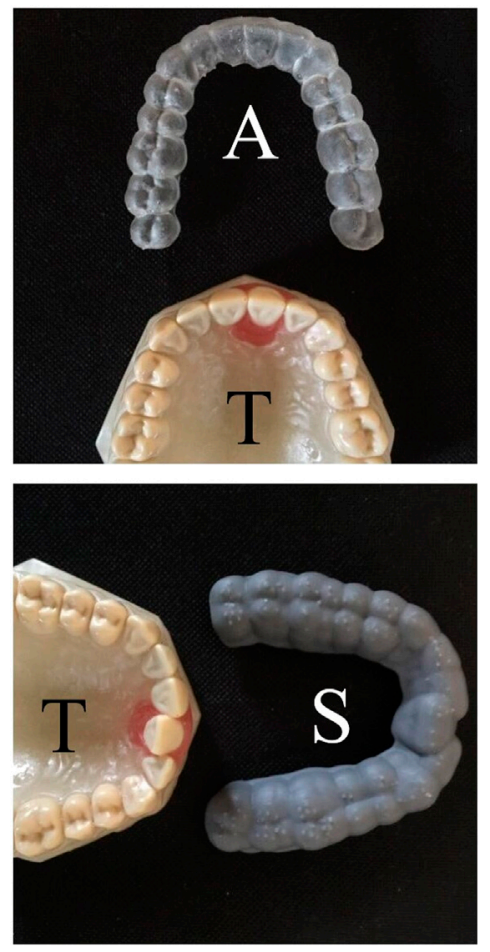

FIGURE 1 | A custom-made typodont (T), a 4D-printed aligner (A), and a gray splint (S).

Copenhagen, Denmark). The model was segmented using an Ortho System software (Ortho Analyzer; 3Shape). Using the appliance designer software (Appliance Designer; 3Shape), two groups of aligners were designed with thicknesses of 0.8 and $1.0 \mathrm{~mm}$ and an offset of $0.2 \mathrm{~mm}$, on the fully aligned model. The aligner geometries were then sent for 3D printing, six aligners per each group $(n=6)$. ClearX v.1.1 3D-printed material (KlineEurope, Dusseldorf, Germany) was used, reported by the suppliers to have shape memory properties. Afterward, tooth 21 was moved palatally in the software model with a total malalignment of $3 \mathrm{~mm}$. The malaligned model was exported as an STL file and was 3D printed using a 3D-printable resin (Dentona Optiprint model; Dentona AG, Dortmund, Germany). A 3D printer (Asiga Max; SCHEU-DENTAL $\mathrm{GmbH}$, Iserlohn, Germany) was used for the previous printing steps, with $62-\mu \mathrm{m}$ high-definition print precision.

Three gray splints of thickness $3.0 \mathrm{~mm}$ (Figure 1) were designed over the malaligned model and were 3D printed (Form 3BL Basic Package; Formlabs, Somerville, MA, USA) using a resin material (Grey Resin $1 \mathrm{~L}$; Formlabs). The first splint was produced over the malaligned model. It was used as an "index" to reposition the typodont (tooth 21) to the exact same malaligned position during testing. The second and third splints were produced over the $3 \mathrm{D}$ aligners, one for each different thickness. They were used for reforming and adaptation of the softened aligners to the malaligned models before testing.

The adaptation process was done by immersing the aligner in a hot water bath of $80^{\circ} \mathrm{C}$ for $30 \mathrm{~s}$ to ensure exceeding the glass transition temperature ( $\mathrm{Tg}$ ) of the material, reported by the supplier to be at $30^{\circ} \mathrm{C}$, and to ensure enough softening of the material. The aligner was then adapted on the malaligned model by the aid of the corresponding adaptor splint. They were then let together to cool down in a cold water bath of $5^{\circ} \mathrm{C}$ so that the aligner can maintain its new malaligned shape (malaligned aligner).

The wax around tooth 21 of the typodont model was softened, and the index splint was used to reposition tooth 21 in the typodont model at 3-mm malposition. The typodont was then placed in a $5^{\circ} \mathrm{C}$ water bath for $10 \mathrm{~min}$ to ensure that the wax was no longer soft and could withstand aligner placement without getting distorted. The malaligned typodont model was then scanned (scan 1). Afterward, the malaligned aligner was placed on the malaligned typodont, and both were immersed together for $5 \mathrm{~min}$ in a hot water bath of $55^{\circ} \mathrm{C}$, which is the activation temperature recommended by the supplier. This initiated the shape memory recovery of the aligner and softened the wax around tooth 21 in the typodont to allow the correction movement of the tooth to happen by the memory recovery of the SMP aligner. Subsequently, they were taken out from the hot water bath and put back in a cold water bath $\left(5^{\circ} \mathrm{C}\right)$, so that the wax could get hard at the new position before removing the aligner to avoid distortion. The typodont model was then scanned again (scan 2). Scan 2 was superimposed with scan 1, and the correction was measured by the Ortho Analyzer software (Figure 2). The test was repeated six times for each group $(0.8$ and $1.0 \mathrm{~mm})$, each time new wax and new aligner were used. Using this test, the aligner shape recovery was recorded visually. A schematic diagram illustrating the main steps of ClearX 4D aligner method was added (Figure 3).

\section{3-PB Test}

The experimental investigation of the force systems generated was performed by a custom-made biomechanical system (OMSS; Drescher et al., 1991) at the University Hospital Bonn in Germany. Six 3D-printed specimens of the $4 \mathrm{D}$ ClearX v.1.1 material were produced in dimensions $\left(50 \times 10 \times 1.0 \mathrm{~mm}^{3}\right)$ and mounted in the biomechanical testing machine (OMSS, Figure 4) to perform a 2-mm vertical 3-PB tests under standardized conditions at a rate of $5 \mathrm{~mm}$ per minute and at different temperatures of $20^{\circ} \mathrm{C}, 30^{\circ} \mathrm{C}, 35^{\circ} \mathrm{C}, 37^{\circ} \mathrm{C}, 40^{\circ} \mathrm{C}$, and $50^{\circ} \mathrm{C}$. The force/deflection curves were recorded, and the maximum

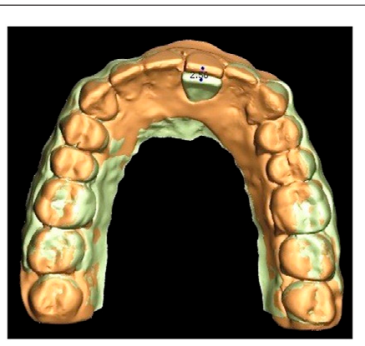

FIGURE 2 | Superimposition of the typodont scans before and after testing of the 4D aligner, scan 1 (before, green) and scan 2 (after, orange). 


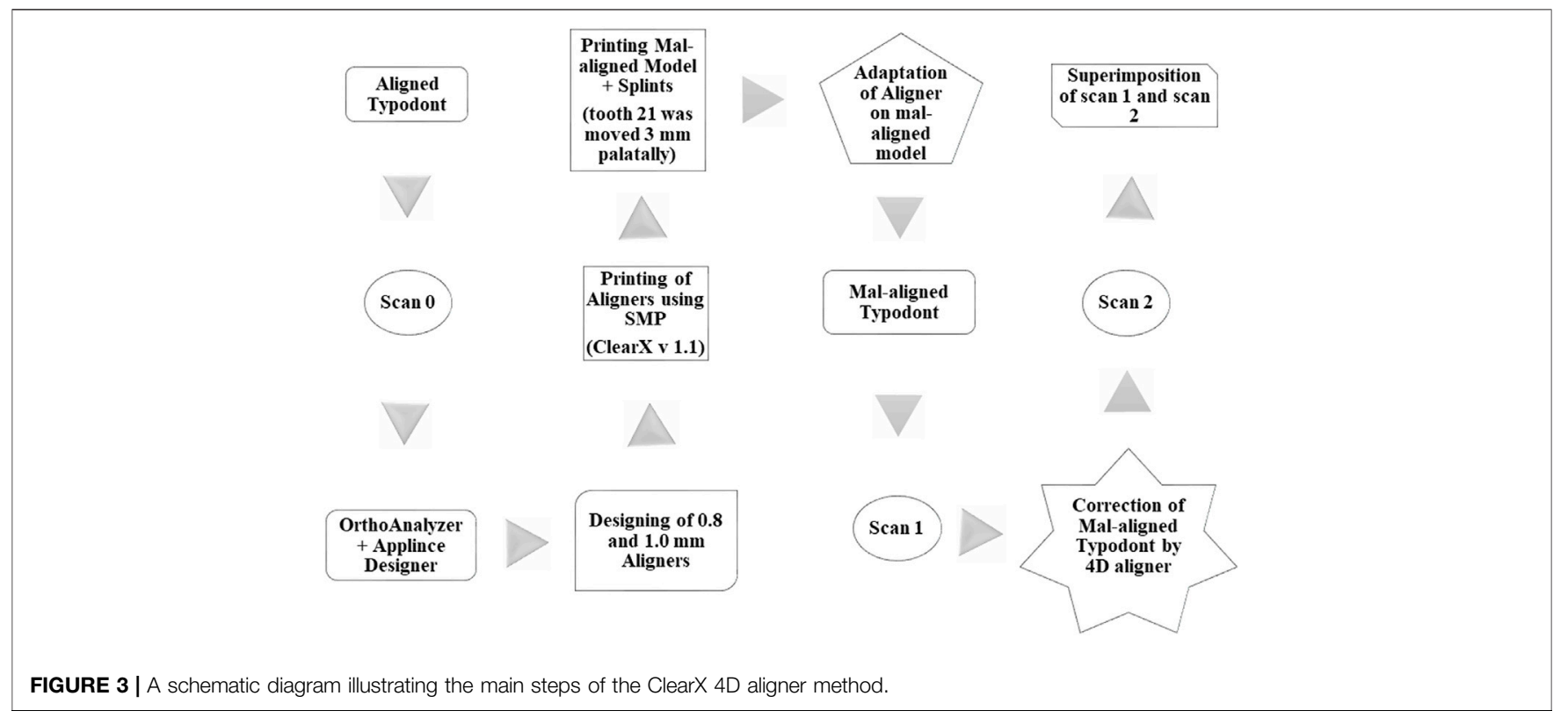

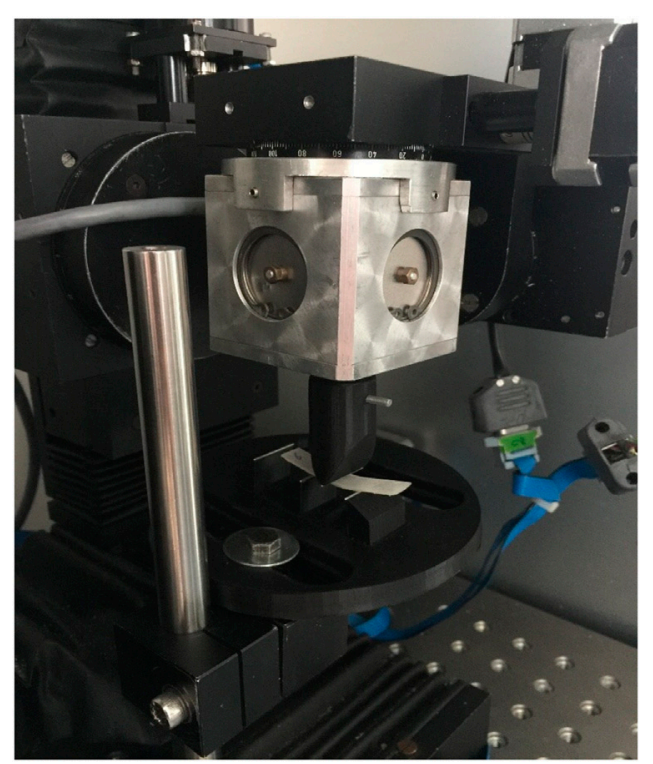

FIGURE 4 | Three-point bending test of a 4D-printed specimen using the orthodontic measurement and simulation system (OMSS) at different temperatures.

generated force was determined. Each cycle was repeated two times with the same specimen.

\section{Experimental Simulation of Tooth \\ Movement}

The typodont without the movable tooth 21 was duplicated using Technovit 4004, and tooth 21 was separated. The resin replica was mounted into OMSS (Figure 5). In several articles (Drescher

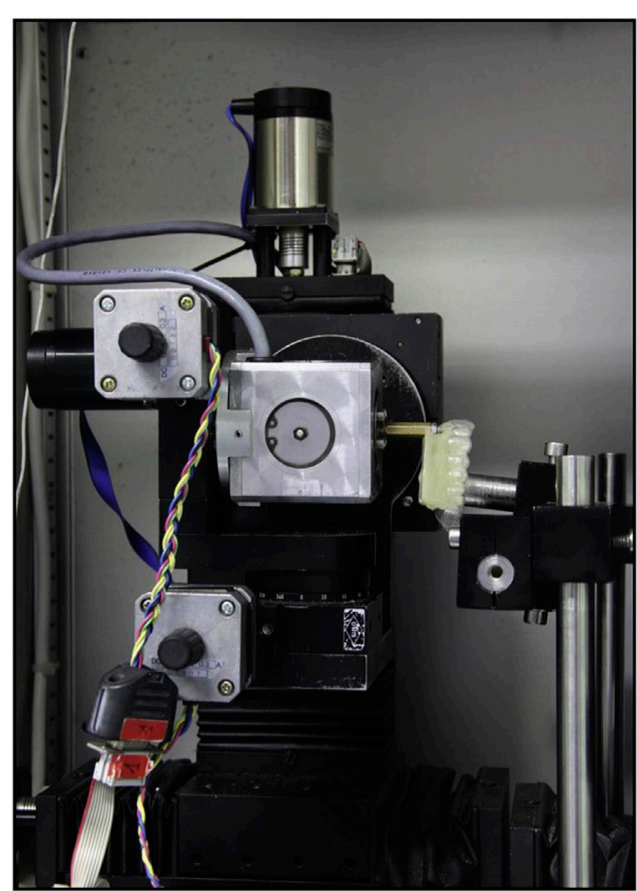

FIGURE 5 |Orthodontic measurement and simulation system (OMSS): a resin replica with a 4D-printed aligner is mounted in the system. Tooth 21 to be analyzed was connected to a computer-controlled 3D sensor. The measured moments and forces are registered, and the motor-driven positioning table simulates the analyzed tooth movement.

et al., 1991; Simon et al., 2014a), more details of the technical specifications and the software running the experiments of OMSS can be found. The OMSS device consists of sensors that can be used for measuring force and moment vectors in three 
TABLE 1 | Mean \pm standard deviation (SD) of translation and forces conducted on upper central incisor (tooth 21) by 4D-printed aligners of two thicknesses

\begin{tabular}{|c|c|c|c|c|c|}
\hline & \multirow[t]{2}{*}{ Thickness (mm) } & \multicolumn{3}{|c|}{ Temperature } & \multirow[t]{2}{*}{$p$ value } \\
\hline & & $37^{\circ} \mathrm{C}$ & $45^{\circ} \mathrm{C}$ & $55^{\circ} \mathrm{C}$ & \\
\hline Translation (mean \pm SD) & $\begin{array}{l}0.8 \\
1.0\end{array}$ & $\begin{array}{c}1.0 \pm 0.4^{\mathrm{Ba}} \\
1.1 \pm 0.4^{\mathrm{Ba}} \\
0.701 \mathrm{~ns}\end{array}$ & $\begin{array}{c}2.4 \pm 0.6^{\mathrm{Aa}} \\
1.6 \pm 0.2^{\mathrm{Bb}} \\
0.004\end{array}$ & $\begin{array}{c}2.1 \pm 0.5^{\mathrm{Ab}} \\
2.8 \pm 0.1^{\mathrm{Aa}} \\
0.006\end{array}$ & $\begin{array}{l}<0.001^{*} \\
<0.001^{*}\end{array}$ \\
\hline Force (mean $\pm \mathrm{SD}$ ) & $\begin{array}{l}0.8 \\
1.0\end{array}$ & $\begin{array}{c}0.3 \pm 0.1^{\mathrm{Ba}} \\
0.3 \pm 0.1^{\mathrm{Ba}} \\
0.701 \mathrm{~ns}\end{array}$ & $\begin{array}{c}0.2 \pm 0.1^{\mathrm{Ba}} \\
0.3 \pm 0.1^{\mathrm{Ba}} \\
0.701 \mathrm{~ns}\end{array}$ & $\begin{array}{c}0.7 \pm 0.2^{\mathrm{Aa}} \\
0.6 \pm 0.2^{\mathrm{Aa}} \\
0.701 \mathrm{~ns}\end{array}$ & $\begin{array}{l}<0.001^{*} \\
<0.001^{*}\end{array}$ \\
\hline
\end{tabular}

Measurement was done at different temperatures in a custom-made biomechanical system (OMSS). Different uppercase and lowercase superscript letters indicate a statistically significant difference within the same horizontal row and vertical columns, respectively.

*Significant ( $\mathrm{p} \leq 0.05)$. ns, nonsignificant $(\mathrm{p}>0.05)$.

dimensions $(x, y$, and $z)$. Sensors are mounted on a 6-axes motordriven positioning table, which is able to perform full $3 \mathrm{D}$ movements. To describe tooth movements in all three spatial dimensions, a coordinate system was set up. The positive $x$ axis $(+X)$ describes extrusive and the negative $x$ axis $(-X)$ describes intrusive movements and forces, parallel to the long axis of the tooth. Horizontal forces and movements are described on the $y$ axis and the $z$ axis, where $+Z$ represented the facial movement and force, $-Z$ represented lingual movement and force, $+Y$ represented distal movement and force, and $-Y$ represented mesial movement and force.

The separated tooth 21 was connected to a sensor. Adjustments were made with the index splint so that tooth 21 was passively set in its $3-\mathrm{mm}$ lingual malposition in respect to the other teeth of the resin replica. The whole apparatus was enclosed in a temperature-controlled chamber in order to run the test at different temperatures. Six new aligners for each group of two thicknesses $(0.8$ and $1.0 \mathrm{~mm})$ were $3 \mathrm{D}$ printed on the aligned model. Each aligner was reformed on the malaligned position as described previously at the typodont experiment. The malaligned aligner was mounted on the resin replica, in a way that almost no active forces (zero forces) were transferred to the tooth, whereas the thermal chamber of OMSS is at room temperature $\left(20^{\circ} \mathrm{C}\right.$, below $\mathrm{Tg}$ ). The temperature of the chamber was increased to $37^{\circ} \mathrm{C}, 45^{\circ} \mathrm{C}$, and $55^{\circ} \mathrm{C}$. By means of continuous measurements of the force systems and simulation of the resulting movement of tooth 21 , the force progression generated by an aligner was measured, and the experimentally resulting tooth movement was calculated. For tooth movements in the three dimensions, the measurements were terminated when forces decayed below $0.02 \mathrm{~N}$.

\section{Statistical Analysis}

Independent $t$ test was used to compare movement of the typodont tooth with different thicknesses of the aligner. Twoway analysis of variance followed by Bonferroni correction was used to study the effect of different tested variables (temperature and thickness of the aligner) and their interaction on force and translation. The significance level was set at $p \leq 0.05$ within all tests. Statistical analysis was performed using IBM SPSS Statistics version 25 for Windows (IBM Company, Endicott, NY, USA).
TABLE 2 | Mean \pm standard deviation (SD) of translation of upper central incisor (tooth 21) by 4D-printed aligners of two thicknesses

Translation (mean \pm SD)

$p$ value (ns)

\begin{tabular}{lccc}
\hline Thickness & Typodont & OMSS & \\
\hline $0.8 \mathrm{~mm}$ & $2.2 \pm 0.5^{\mathrm{Aa}}$ & $2.1 \pm 0.5^{\mathrm{Ab}}$ & $0.617^{\star}$ \\
$1.0 \mathrm{~mm}$ & $2.6 \pm 0.5^{\mathrm{Aa}}$ & $2.8 \pm 0.1^{\mathrm{Aa}}$ & $0.257^{\star}$ \\
$p$ value & $0.152 \mathrm{~ns}$ & $0.006^{\star}$ &
\end{tabular}

Measurement was done at temperature of $55^{\circ} \mathrm{C}$ on a typodont model and in a custommade biomechanical system (OMSS). Different uppercase and lowercase superscript letters indicate a statistically significant difference within the same horizontal row and vertical columns, respectively.

${ }^{*}$ Significant $(\mathrm{p} \leq 0.05)$. ns, nonsignificant $(\mathrm{p}>0.05)$.

\section{RESULTS}

On the typodont, 4D-printed aligners succeeded to achieve a significant tooth movement $(2.5 \pm 0.5 \mathrm{~mm})$, with insignificant difference between the different thicknesses (Figure 2 and Table 2). In 3-PB test, the maximum forces measured at $20^{\circ} \mathrm{C}$, $30^{\circ} \mathrm{C}, 37^{\circ} \mathrm{C}, 45^{\circ} \mathrm{C}$, and $55^{\circ} \mathrm{C}$ were $3.8 \pm 1.1,2.5 \pm 0.9,1.7 \pm 0.6,1.0 \pm$ 0.4 , and $0.5 \pm 0.4 \mathrm{~N}$, respectively (Figure 6).

In OMSS simulations, the forces delivered on palatal displacement of tooth 21 at $37^{\circ} \mathrm{C}, 45^{\circ} \mathrm{C}$, and $55^{\circ} \mathrm{C}$ by 0.8 - $\mathrm{mm}$ aligners were $0.3 \pm 0.1$, $0.2 \pm 0.1$, and $0.7 \pm 0.2 \mathrm{~N}$, respectively, whereas those by $1.0-\mathrm{mm}$ aligners were $0.3 \pm 0.1,0.3 \pm 0.1$, and $0.6 \pm 0.2 \mathrm{~N}$, respectively (Figure 7 and Table 1). Varying degrees of temperature had a significant effect on the force, whereas the thickness of the aligner along with its interaction with temperature had no significant effect. Pairwise comparison showed that aligner subjected to $55^{\circ} \mathrm{C}$ conducted a significantly higher force than those subjected to $37^{\circ} \mathrm{C}$ and $45^{\circ} \mathrm{C}$. A good concordance with movement of tooth 21 in the typodont experiment could be shown in OMSS with insignificant difference between them (Table 2). At $55^{\circ} \mathrm{C}$, there was a higher significant translation by $1.0-\mathrm{mm}$ aligner than the $0.8-\mathrm{mm}$ one (Tables 1 and 2).

\section{DISCUSSION}

Although orthodontic aligners have been studied in several aspects and great progress was done in orthodontic treatment by aligners (Bowman, 2017), practitioners still report some 

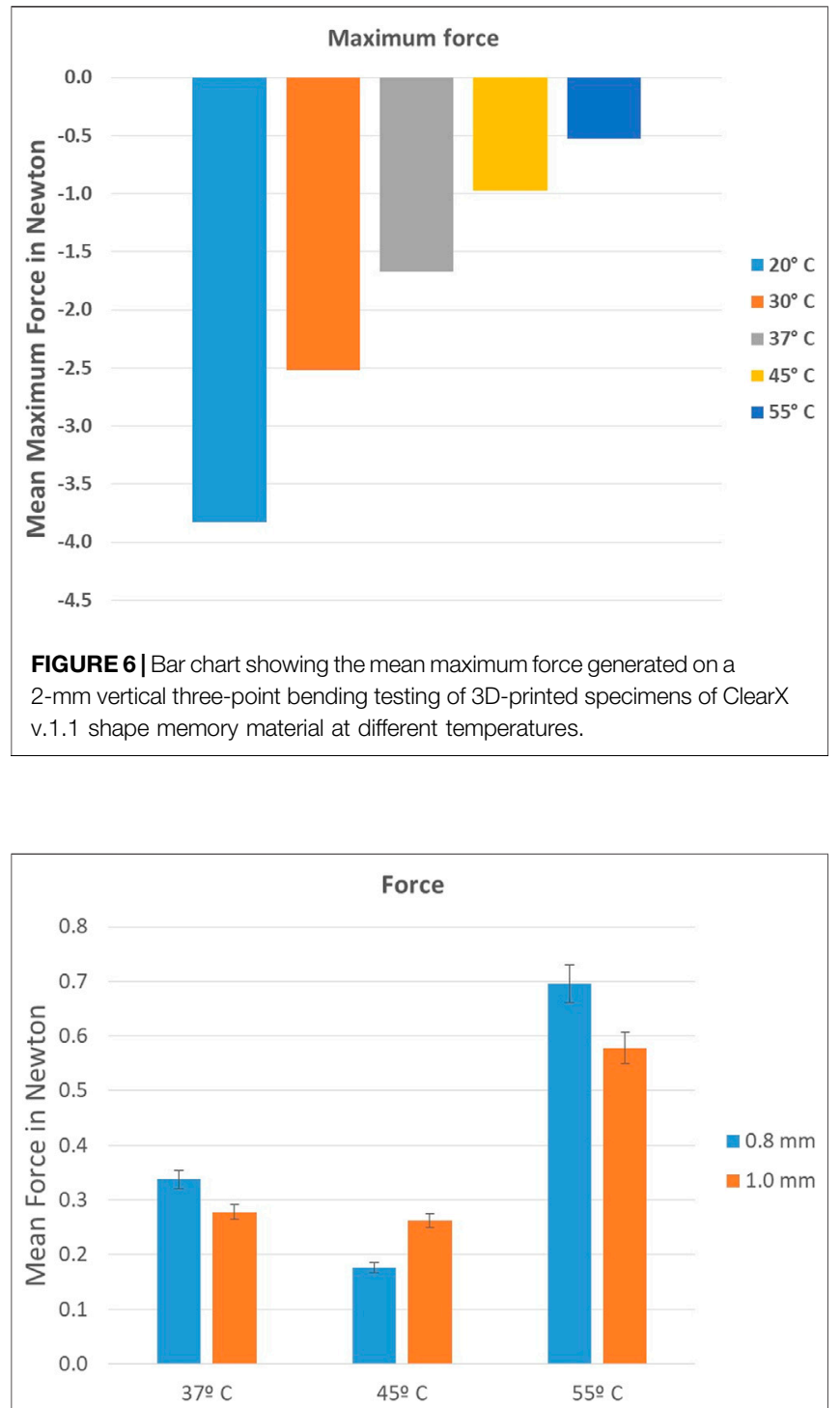

FIGURE 7 | Bar chart showing forces generated by 4D-printed aligners of two different thicknesses; measurement was done at different temperatures in a custom-made biomechanical system (OMSS).

drawbacks to aligner use. On the top of these drawbacks are the limited movement achieved by each single aligner, which leads to use of changing regime through a large series of aligners per treatment (Simon et al., 2014b; Elshazly et al., 2021). Therefore, we believe that if a method could be applied to decrease the number of the aligners per treatment, together with a method to accelerate the biological movement of the teeth, that would be a quantum leap in the field of orthodontics.

The introduction of new aligner materials draws the attention of many researchers. Some of them introduced smart materials, particularly SMPs (Silverman and Cohen, 1969; Choi and Kim, 2005; Lai and Rule, 2020; Elshazly et al., 2021). The idea of clear aligners made of SMPs is aiming to reduce the cost and time of the treatment by using a dynamic aligner that can change its shape intentionally over treatment time, and consequently one shape memory aligner could substitute a series of conventional aligners and overcome the stepwise system. Furthermore, it helps to decrease the plastic consumption by decreasing the number of plastic aligners per treatment plan and thus raise concerns about the ethical responsibility toward the environment (Elshazly et al., 2021). Despite many tryouts mentioned before (Choi and Kim, 2005; Jung and Cho, 2010; Lai and Rule, 2020; Elshazly et al., 2021), still there is a great lack of data in the literature about clear aligners made from SMPs. So far, it is difficult, biomechanicalwise and biocompatibility-wise, to favor an appropriate material.

Thermoresponsive SMPs are a subcategory of smart polymers, which have a novel capacity, to recover their original shape after being deformed, upon specific thermal initiation (Zhang et al., 2009; Meng and Li, 2013). At the molecular level, polymer network-based SMPs consist of at least two different segments, hard and soft, with two different glass transition temperatures (Tg) (Lendlein and Langer, 2002). Shape memory mechanism is attributed to the cooperation of the hard and the soft segments. Phase separation is essential in this mechanism. The separation occurs only above a threshold temperature (transition temperature); prior to it, no phase separation occurs. Soft segments act as a switch (matrix phase) responsible for shape changing, and hard segments act as cross-links responsible for preserving the original shape (Lendlein and Kelch, 2002; Behl and Lendlein, 2007; Zhang et al., 2009). Many of SMPs have a Tg near the body temperature; thus, the body temperature could act as an initiator for shape memory change (Gorna and Gogolewski, 2003). Moreover, many SMPs have the advantage of possessing several inherent properties, such as transparency, low density, and reduced cost (Jung and Cho, 2010). In addition, the shape recovery of some SMPs could last up to approximately 3 months, which might be suitable for orthodontic applications (Small et al., 2010). These advantages may qualify them to be introduced in the fabrication of orthodontic appliances for treatment of initial alignment and leveling of aesthetically concerned patients (Nakasima et al., 1991), as well as the correction of malaligned and severely rotated teeth (Mahmood et al., 2019).

On planning of an orthodontic treatment with an aligner system, it is important to make a biomechanical analysis of the used material and the appliance and know the exact distribution of the forces and moments. The flexure modulus is a mechanical property that relates stress to strain in flexural deformation, and it is an indicator of the material's tendency to resist bending. The developed force is directly proportional to the flexure modulus and to the third order of the thickness in bending direction (Zweben et al., 1979). In orthodontic appliances such as aligners, it is an indicator of the effectiveness for tooth movement (Ryu et al., 2018). The 3-PB test is a method used to determine the flexure modulus and the maximum force delivered upon deflection (Kwon et al., 2008; Min et al., 2010). Additionally, to know the exact force systems of the aligner in all three planes of space, an OMSS was used (Drescher et al., 1991; Bourauel et al., 1992). Moreover, based on the force system, the control program of OMSS calculated the developed tooth movement by using a mathematical model, taking into consideration the center of resistance of the measured tooth (Pedersen et al., 1990). 
Optimal force delivery is a must for an ideal orthodontic treatment, in order to achieve a maximum rate of tooth movement without causing irreversible damage to the biological tissues (Kwon et al., 2008). A light continuous force is required for ideal tooth movement. In case of excessive force to a target tooth, indirect bone resorption can occur; accordingly, the speed of tooth movement will be slower, or root resorption may occur. On the contrary, if sufficient force is not delivered to a target tooth, tooth movement will not be obtained (Proffit et al., 2000). Consequently, optimal forces are important for ideal tooth movement. For instance, the optimal forces for tipping movement of a single tooth were reported to be from 0.50 to $0.75 \mathrm{~N}$, for rotation control are $1.00-1.50 \mathrm{~N}$, for torque control are $0.75-1.25 \mathrm{~N}$, and for bodily movement are $0.75-1.25 \mathrm{~N}$ (Proffit et al., 2000; Kwon et al., 2008). In conventional aligner systems, the force delivery to a target tooth is generated by the tendency of the plastic material through its resiliency to return to its resting state instead of being deformed $0.25-0.33 \mathrm{~mm}$ in some systems (Simon et al., 2014a) or 0.50-1.00 mm in other systems (Sheridan et al., 1993). However, in the 4D-printed aligner system, the aligners are made of SMPs, and the force delivery is obtained from the shape recovery of the material upon appropriate stimulations (Elshazly et al., 2021). Additionally, the 3D printing of the material gives advantage of avoiding the significant decrease of mechanical properties after deep drawing of thermoformed material (Ryu et al., 2018).

The technology of $4 \mathrm{D}$ printing is based on the $3 \mathrm{D}$ printing of SMPs. In our study, a 3D-printable material (ClearX v.1.1), reported by the supplier to possess thermoresponsive shape memory properties, was used in fabrication of orthodontic clear aligners (4D aligner). A typodont model was used in the study as an initial proof of the movement of the tooth, similar to many other reports (Ishida et al., 2020; Elshazly et al., 2021; Ho et al., 2021). A simple orthodontic case was used in the study, where only a bodily movement of one tooth (maxillary upper incisor, tooth 21) was tested, whereas the other teeth remained fixed in the resin and were used to provide retention to the whole aligner. The maximum forces generated on $2-\mathrm{mm}$ deflection at different temperatures were measured. Furthermore, the forces generated from shape recovery, which should be responsible for the tooth movement, were measured by a custom-made biomechanical system (OMSS).

The results show success of the shape memory aligner to achieve a significant movement of tooth 21 on the typodont, and a congruent movement was also measured in OMSS. The targeted alignment movement $(3.00 \mathrm{~mm})$ was not reached; only $2.06-2.82 \mathrm{~mm}$ was achieved, which could be referred to the resistance of the wax (in typodont test) and the rigidity of the sensor (in OMSS), in front of the low forces generated by the shape recovery. Nevertheless, the results are satisfactory, and the achieved movement by one shape memory aligner equals nearly the movement that could be achieved by 10 conventional aligners.

In the current study, the force delivery upon deflection of the aligner by bodily movement of tooth 21 for $2 \mathrm{~mm}$ needed to be simulated; therefore, the span length in the 3-PB test (distance between the two supports) was set at $24 \mathrm{~mm}$, which was equal to the sum of the average widths of the two maxillary central incisors and one lateral incisor, and the specimen was deflected vertically at its center to a maximum of $2 \mathrm{~mm}$ (Kwon et al., 2008). The results of our study (Figure 7 ) show that the range of maximum force delivered on a $2-\mathrm{mm}$ deflection at temperatures from $37^{\circ} \mathrm{C}$ to $55^{\circ} \mathrm{C}$ (the activation temperature range of the material) with sheet thickness of $1 \mathrm{~mm}$ ranged from 1.20 to $0.50 \mathrm{~N}$. This is located within the acceptable range of orthodontic forces, and it is in the same range of forces generated by conventional aligners upon deflections of 0.20-0.50 mm (Proffit et al., 2000; Kwon et al., 2008). The results show also that the maximum forces are decreasing with increasing the temperature, which can be explained by the fact that the heat in such thermoplastic material leads to a type of softening through debonding of the secondary bonds of the cross-links of the material and therefore weakens the material.

The generated forces at different temperatures $\left(37^{\circ} \mathrm{C}, 45^{\circ} \mathrm{C}\right.$, $\left.55^{\circ} \mathrm{C}\right)$ measured by OMSS $(0.30-0.70 \mathrm{~N})$ are also in the range of acceptable physiological orthodontic forces reported by many studies (Hahn et al., 2010; Simon et al., 2014a; Elkholy et al., 2016; Elkholy et al., 2017; Iliadi et al., 2019). Moreover, these findings are supported by a study by Nakasima et al. (1991), in which it was reported that SMPs are able, at properly controlled oral conditions, to produce light long-lasting forces. They used a stretched orthodontic wire of polynorbornene, a type of SMP, and showed that it could reverse gradually to its original shape upon heating at a temperature of $50^{\circ} \mathrm{C}$ and generate forces in the physiological range, which could be used to move a human tooth.

In our study, the forces generated at $50^{\circ} \mathrm{C}$ were higher than the forces generated at $37^{\circ} \mathrm{C}$, yet in the accepted range. Interestingly, there was a reduction of forces at $45^{\circ} \mathrm{C}$ in comparison to the forces at $37^{\circ} \mathrm{C}$ and $55^{\circ} \mathrm{C}$. Explanation of such situation, especially in a viscoelastic material such as the tested material, may need further investigation of the material, such as dynamic thermomechanical analysis and differential scanning calorimetry, in order to get a more clear picture of the phase transition at different temperatures.

Contradicting with previous studies (Kwon et al., 2008; Hahn et al., 2009; Min et al., 2010; Ryu et al., 2018), the current study reported that using thicker aligners has no significant effect on the generated force, except at $55^{\circ} \mathrm{C}$ in the OMSS. That could be due to inaccuracy of the $3 \mathrm{D}$ printing in a way that the aligner thickness is not homogenous, thicker in parts and thinner in others. However, the findings are, from other prospective, generally in agreement with Nam and Pei (2020), who revealed that the shape memory effect of $4 \mathrm{D}$-printed parts is mostly influenced by the recovery temperature and the deformation temperature.

This study still has many limitations. We measured forces for an isolated experimental movement of a single tooth, which is a simplified model that cannot reflect the more complex clinical cases, in which several teeth are included in the treatment plan. Also, we did not fully consider the intraoral conditions such as salivation and humidity, especially that some polymers are sensitive to moisture due to presence of hydrogen bonding between the polymeric chains (Yen et al., 1991; McKiernan et al., 2002). Additionally, the OMSS enables both measurements of the forces of the initial situation and the dynamic force progression during tooth movement; however, 
in the setup of the present study, sensor and tooth were connected rigidly, with the limitation of simulation of some clinical parameters such as periodontal ligament (PDL), mastication, as well as soft-tissue reactions (Bourauel et al., 1992). Besides, OMSS is based on the hypothesis that there is a linear relationship between the speed of tooth movement and the amount of applied force, which could not really simulate the biomechanical behavior of PDL. Moreover, an ideal center of resistance was used for each tooth (Simon et al., 2014a).

Further studies on the mechanical and physical properties of the used SMP materials should be performed. More clinically oriented simulated models for the force delivery by SMP materials should be developed. Moreover, it is still a challenge to introduce a clinically applicable technique to use the shape memory aligners.

\section{CONCLUSION}

Initial investigation of a 3D-printed aligner made of SMPs (4D aligner) shows its capability of moving teeth by biocompatible orthodontic forces after a suitable thermal stimulus within the oral temperature range.

\section{REFERENCES}

Behl, M., and Lendlein, A. (2007). Shape-memory Polymers. Mater. Today 10, 20-28. doi:10.1016/s1369-7021(07)70047-0

Bourauel, C., Drescher, D., and Thier, M. (1992). An Experimental Apparatus for the Simulation of Three-Dimensional Movements in Orthodontics. J. Biomed. Eng. 14, 371-378. doi:10.1016/0141-5425(92)90081-u

Bowman, S. J. (2017). Improving the Predictability of clear Aligners. Semin. Orthod. 23, 65-75. doi:10.1053/j.sodo.2016.10.005

Boyd, R. L., and Waskalic, V. (2001). Three-dimensional Diagnosis Andorthodontic Treatment of Complex Malocclusions with the Invisalign Appliance. Semin. Orthod. 7, 274-293. doi:10.1053/ sodo.2001.25414

Choi, Y., and Kim, K.-T. (2005). Orthodontic Appliance by Using a Shape Memory Polymer. South Korean Patent No KR100554713B1.

Drescher, D., Bourauel, C., and Thier, M. (1991). Application of the Orthodontic Measurement and Simulation System (OMSS) in Orthodontics. Eur. J. Orthod. 13, 169-178. doi:10.1093/ejo/13.3.169

Elkholy, F., Schmidt, F., Jäger, R., and Lapatki, B. G. (2017). Forces and Moments Applied during Derotation of a Maxillary central Incisor with Thinner Aligners: an In-Vitro Study. Am. J. Orthod. Dentofacial Orthopedics 151, 407-415. doi:10.1016/j.ajodo.2016.08.020

Elkholy, F., Schmidt, F., Jäger, R., and Lapatki, B. G. (2016). Forces and Moments Delivered by Novel, Thinner PET-G Aligners during Labiopalatal Bodily Movement of a Maxillary central Incisor: An In Vitro Study. Angle Orthod. 86, 883-890. doi:10.2319/011316-37r.1

Elshazly, T. M., Keilig, L., Alkabani, Y., Ghoneima, A., Abuzayda, M., Talaat, S., et al. (2021). Primary Evaluation of Shape Recovery of Orthodontic Aligners Fabricated from Shape Memory Polymer (A Typodont Study). Dentistry J. 9 (3), 31. doi:10.3390/dj9030031

Ercoli, F., Tepedino, M., Parziale, V., and Luzi, C. (2014). A Comparative Study of Two Different clear Aligner Systems. Prog. Orthod. 15, 31. doi:10.1186/s40510014-0031-3

Gorna, K., and Gogolewski, S. (2003). The Effect of Gamma Radiation on Molecular Stability and Mechanical Properties of Biodegradable Polyurethanes for Medical Applications. Polym. Degrad. Stab. 79, 465-474. doi:10.1016/s0141-3910(02)00362-2

\section{DATA AVAILABILITY STATEMENT}

The raw data supporting the conclusion of this article will be made available by the authors, without undue reservation.

\section{AUTHOR CONTRIBUTIONS}

Conceptualization: TE. Data curation, analysis, investigation, and methodology: TE and YA. Resources: TE, CB, MA, and AG. Software: TE, CB, and LK. Supervision, validation and visualization: $\mathrm{CB}, \mathrm{AG}, \mathrm{MA}, \mathrm{WT}$, and ST. Writing-original draft: TE. Writing - review and editing: TE, YA, and CB. All authors have read and agreed to the published version of the manuscript.

\section{FUNDING}

This work was supported by a grant (MBRU-AlMahmeed Collaborative Research Award 2019) from Mohammed Bin Rashid University of Medicine and Health Sciences (MBRU), Project No: ALM 1931.

Hahn, W., Dathe, H., Fialka-Fricke, J., Fricke-Zech, S., Zapf, A., KubeinMeesenburg, D., et al. (2009). Influence of Thermoplastic Appliance Thickness on the Magnitude of Force Delivered to a Maxillary central Incisor during Tipping. Am. J. Orthod. Dentofacial Orthop. 136, 12-13. doi:10.1016/j.ajodo.2008.12.015

Hahn, W., Zapf, A., Dathe, H., Fialka-Fricke, J., Fricke-Zech, S., Gruber, R., et al. (2010). Torquing an Upper central Incisor with Aligners-Aacting Forces and Biomechanical Principles. Eur. J. Orthod. 32, 607-613. doi:10.1093/ejo/cjq007

Ho, C.-T., Huang, Y.-T., Chao, C.-W., Huang, T.-H., and Kao, C.-T. (2021). Effects of Different Aligner Materials and Attachments on Orthodontic Behavior. J. Dental Sci. 16, 1001-1009. doi:10.1016/j.jds.2021.01.011

Iliadi, A., Koletsi, D., and Eliades, T. (2019). Forces and Moments Generated by Aligner-type Appliances for Orthodontic Tooth Movement: A Systematic Review and Meta-analysis. Orthod. Craniofac. Res. 22, 248-258. doi:10.1111/ ocr.12333

Ishida, Y., Kuwajima, Y., Lee, C., Ogawa, K., Da Silva, J. D., and Ishikawa-Nagai, S. (2020). Effect of Magnet Position on Tipping and Bodily Tooth Movement in Magnetic Force-Driven Orthodontics. Materials 13 (16), 3588. doi:10.3390/ ma13163588

Jung, Y. C., and Cho, J. W. (2010). Application of Shape Memory Polyurethane in Orthodontic. J. Mater. Sci. Mater. Med. 21, 2881-2886. doi:10.1007/s10856008-3538-7

Kwon, J.-S., Lee, Y.-K., Lim, B.-S., and Lim, Y.-K. (2008). Force Delivery Properties of Thermoplastic Orthodontic Materials. Am. J. Orthod. Dentofacial Orthopedics 133, 228-234. doi:10.1016/j.ajodo.2006.03.034

Lai, M.-L., and Rule, J. D. (2020). Orthodontic Appliance Having Continuous Shape Memory. US Patent No US20200315747A1.

Lendlein, A., and Kelch, S. (2002). Shape-memory Polymers. Angew. Chem. Int. Ed. 41, 2034-2057. doi:10.1002/1521-3773(20020617)41:12<2034:aidanie2034>3.0.co;2-m

Lendlein, A., and Langer, R. (2002). Biodegradable, Elastic Shape-Memory Polymers for Potential Biomedical Applications. Science 296, 1673-1676. doi:10.1126/science.1066102

Mahmood, H. T., Kamal, A. T., Khan, B. N., and Fida, M. (2019). Application of New Biomedical Materials in Orthodontic Appliances. J. Coll. Physicians Surg. Pak 29 (7), 654-657. doi:10.29271/jcpsp.2019.07.654

Martorelli, M., Gerbino, S., Giudice, M., and Ausiello, P. (2013). A Comparison between Customized clear and Removable Orthodontic Appliances 
Manufactured Using RP and CNC Techniques. Dental Mater. 29, e1-e10. doi:10.1016/j.dental.2012.10.011

McKiernan, R. L., Heintz, A. M., Hsu, S. L., Atkins, E. D. T., Penelle, J., and Gido, S. P. (2002). Influence of Hydrogen Bonding on the Crystallization Behavior of Semicrystalline Polyurethanes. Macromolecules 35, 6970-6974. doi:10.1021/ ma0201274

Mehta, F., and Mehta, S. (2014). Aligners: the Rapidly Growing Trend in Orthodontics Around the World. Indian J. Basic Appl. Med. Res. 3, 402-409.

Meng, H., and Li, G. (2013). A Review of Stimuli-Responsive Shape Memory Polymer Composites. Polymer 54, 2199-2221. doi:10.1016/ j.polymer.2013.02.023

Min, S., Hwang, C.-J., Yu, H.-S., Lee, S.-B., and Cha, J.-Y. (2010). The Effect of Thickness and Deflection of Orthodontic Thermoplastic Materials on its Mechanical Properties. Korean J. Orthod. 40, 16-26. doi:10.4041/ kjod.2010.40.1.16

Momtaz, P. (2016). The Effect of Attachment Placement and Location on Rotational Control of Conical Teeth Using Clear Aligner Therapy. Master's thesis/dissertation. Las Vegas(USA): University of Nevada).

Morton, J., Derakhshan, M., Kaza, S., and Li, C. (2017). Design of the Invisalign System Performance. Semin. Orthod. 23, 3-11. doi:10.1053/j.sodo.2016.10.001

Nakasima, A., Hu, J. R., Ichinose, M., and Shimada, H. (1991). Potential Application of Shape Memory Plastic as Elastic Material in Clinical Orthodontics. Eur. J. Orthod. 13, 179-186. doi:10.1093/ejo/13.3.179

Nam, S., and Pei, E. (2020). The Influence of Shape Changing Behaviors from 4D Printing through Material Extrusion Print Patterns and Infill Densities. Materials 13 (17), 3754. doi:10.3390/ma13173754

Ojima, K., and Kau, C. H. (2017). A Perspective in Accelerated Orthodontics with Aligner Treatment. Semin. Orthod. 23, 76-82. doi:10.1053/j.sodo.2016.10.002

Pedersen, E., Andersen, K., and Gjessing, P. E. (1990). Electronic Determination of Centres of Rotation Produced by Orthodontic Force Systems. Eur. J. Orthod. 12, 272-280. doi:10.1093/ejo/12.3.272

Pei, E., Loh, G. H., and Nam, S. (2020). Concepts and Terminologies in 4D Printing. Appl. Sci. 10 (13), 4443. doi:10.3390/app10134443

Phan, X., and Ling, P. H. (2007). Clinical Limitations of Invisalign. J. Can. Dent Assoc. 73 (3), 263-266.

Proffit, W. R., Fields, H. W., Sarver, D. M., and Ackerman, J. L. (2000). Contemporary Orthodontics. St. Louis: Mosby.

Ryu, J.-H., Kwon, J.-S., Jiang, H. B., Cha, J.-Y., and Kim, K.-M. (2018). Effects of Thermoforming on the Physical and Mechanical Properties of Thermoplastic Materials for Transparent Orthodontic Aligners. Korean J. Orthod. 48, 316-325. doi:10.4041/kjod.2018.48.5.316

Sheridan, J. J., LeDoux, W., and McMinn, R. (1993). Essix Retainers: Fabrication and Supervision for Permanent Retention. J. Clin. Orthod. 27, 37-45.

Silverman, E., and Cohen, M. (1969). Orthodontic Appliance. US Patent No US3421221A.
Simon, M., Keilig, L., Schwarze, J., Jung, B. A., and Bourauel, C. (2014). Treatment Outcome and Efficacy of an Aligner Technique-Rregarding Incisor Torque, Premolar Derotation and Molar Distalization. BMC Oral Health 14, 1-7. doi:10.1186/1472-6831-14-68

Simon, M., Keilig, L., Schwarze, J., Jung, B. A., and Bourauel, C. (2014). Forces and Moments Generated by Removable Thermoplastic Aligners: Incisor Torque, Premolar Derotation, and Molar Distalization. Am. J. Orthod. Dentofacial Orthopedics 145, 728-736. doi:10.1016/j.ajodo.2014.03.015

Small, IV, W., Singhal, P., Wilson, T. S., and Maitland, D. J. (2010). Biomedical Applications of Thermally Activated Shape Memory Polymers. J. Mater. Chem. 20, 3356-3366. doi:10.1039/b923717h

Tamer, I., Öztaş, E., Oztas, E., and Marsan, G. (2019). Orthodontic Treatment with Clear Aligners and the Scientific Reality behind Their Marketing: A Literature Review. Turk J. Orthod. 32 (4), 241-246. doi:10.5152/turkjorthod.2019.18083

Thukral, R., and Gupta, A. (2015). Invisalign: Invisible Orthodontic Treatment-A Review. J. Adv. Med. Dent. Sci. Res. 3 (5), 42-44.

Yen, F.-S., Lin, L.-L., and Hong, J.-L. (1991). Hydrogen-bond Interactions between Urethane- Urethane and Urethane- Ester Linkages in a Liquid Crystalline Poly (Ester- Urethane). Macromolecules 32, 3068-3079.

Zhang, H., Wang, H., Zhong, W., and Du, Q. (2009). A Novel Type of Shape Memory Polymer Blend and the Shape Memory Mechanism. Polymer 50, 1596-1601. doi:10.1016/j.polymer.2009.01.011

Zweben, C., Smith, W. S., and Wardle, M. W. (1979). "Test Methods for Fiber Tensile Strength, Composite Flexural Modulus, and Properties of FabricReinforced Laminates," in Composite Materials: Testing and Design (Fifth Conference). Editor S. Tsai (West Conshohocken, PA: ASTM International), 228-262.

Conflict of Interest: The authors declare that the research was conducted in the absence of any commercial or financial relationships that could be construed as a potential conflict of interest.

Publisher's Note: All claims expressed in this article are solely those of the authors and do not necessarily represent those of their affiliated organizations, or those of the publisher, the editors and the reviewers. Any product that may be evaluated in this article, or claim that may be made by its manufacturer, is not guaranteed or endorsed by the publisher.

Copyright $\odot 2022$ Elshazly, Keilig, Alkabani, Ghoneima, Abuzayda, Talaat, Talaat and Bourauel. This is an open-access article distributed under the terms of the Creative Commons Attribution License (CC BY). The use, distribution or reproduction in other forums is permitted, provided the original author $(s)$ and the copyright owner(s) are credited and that the original publication in this journal is cited, in accordance with accepted academic practice. No use, distribution or reproduction is permitted which does not comply with these terms. 